\title{
Platelet-Function Testing in Patients Undergoing Neurovascular Procedures: Caught between a Rock and a Hard Place
}

\author{
J. Comin and D.F. Kallmes
}

\begin{abstract}
SUMMARY: In the context of neurointerventional procedures, clopidogrel hyper-responsiveness has been associated with hemorrhage; on the other hand, clopidogrel resistance has been associated with thromboembolism. This might seem to make a compelling argument in favor of routine platelet testing. Our reading of the literature leads us to conclude that routine platelet testing in neurointerventional procedures is not, unfortunately, ready for prime time.
\end{abstract}

ABBREVIATIONS: CREST = Clopidogrel Effect on Platelet Reactivity in Patients with Stent Thrombosis; GRAVITAS = Gauging Responsiveness with a VerifyNow Assay; OASIS-7 = Optimal Antiplatelet Strategy for Interventions; PCI = percutaneous coronary intervention; POPULAR = Point-of-Care Platelet Function Assays Predict Clinical Outcomes in Clopidogrel pre-treated patients undergoing elective PCl; TRIGGER-PCI = Testing Platelet Reactivity in Patients Undergoing Elective Stent Placement on Clopidogrel to Guide Alternate Therapy with Prasugrel.

T he articles by Goh et $\mathrm{al}^{1}$ and Fifi et $\mathrm{al}^{2}$ in this month's journal highlight the intense scrutiny now focused on platelet-function testing in interventional neuroradiology. The storyline of these 2 bookend articles - on the one hand that clopidogrel hyper-responsiveness was associated with hemorrhage and, on the other hand, that clopidogrel resistance was associated with thromboembolism - seems like compelling evidence in favor of routine platelet testing. However, these articles represent only the tip of a veritable iceberg of literature on the subject of platelet testing in endovascular procedures. If we look below the surface at this iceberg, it becomes clear that similar enthusiasm for platelet testing previously gripped the interventional cardiology community. Both Goh et $\mathrm{al}^{1}$ and Fifi et $\mathrm{al}^{2}$ concluded that further study is needed. It is not clear, however, that such urgent study will result in a consensus regarding platelet testing, as evidenced by the iceberg of literature discussed below. Indeed, our reading of the cardiology literature leads us to the opposite conclusion, specifically that routine platelet testing in neurointerventional procedures is not, unfortunately, ready for prime time.

Both sides of this debate can agree on 3 points regarding platelet function testing for neurovascular intervention:

1) A certain number of patients will experience thrombotic complications from neurovascular interventions, despite being compliant with a standard antiplatelet medication regimen.

From the Department of Radiology (D.F.K.), Mayo Clinic, Rochester, Minnesota; and St Vincent's Hospital Melbourne (J.C.), Fitzroy, Australia.

Please address correspondence to David F. Kallmes, MD, Department of Radiology, 200 First Street SW, Rochester, MN 55905, email: kallmes.david@mayo.edu.

http://dx.doi.org/10.3174/ajnr.A3440
2) High in vitro platelet reactivity predicts an increased incidence of adverse ischemic outcomes in cardiology patients undergoing PCI.

3) Increasing antiplatelet pharmacotherapy reduces this high on-treatment platelet reactivity in vitro.

These points are well-established in the literature, are beyond dispute, and will not be discussed further. However, to justify the use of routine platelet function testing for neurovascular intervention, proponents must make the following assumptions:

1) The increased incidence of adverse ischemic outcomes includes a proportional increase in the incidence of target-vessel thrombosis.

2) The relationship between high in vitro platelet reactivity and adverse outcomes in cardiology patients undergoing PCI can be extrapolated to neurovascular patients.

3) Increasing antiplatelet pharmacotherapy reduces the incidence of adverse thrombotic events in patients with high in vitro platelet reactivity.

4) A reduction in the incidence of adverse thrombotic events could not be achieved more simply, more cheaply, or more effectively in some other way that does not involve platelet function testing.

Unfortunately, there is little evidence to support any of these latter assumptions, and there is some evidence to suggest that they may, in fact, be wrong.

\section{Assumption 1}

The increased incidence of adverse ischemic outcomes includes a proportional increase in the incidence of target-vessel thrombosis. That there is an increased risk of adverse ischemic outcomes in 
patients who exhibit high platelet reactivity despite compliance with an antiplatelet regimen has been established by rigorous and large meta-analysis of the cardiology literature. ${ }^{3}$

More recently, the POPULAR trial ${ }^{4}$ demonstrated that the results of certain platelet function tests, but not others, were correlated with an increased risk of adverse ischemic events, though this relationship was not as strong as previously thought. However, this increased risk applies to a broad set of outcomes, including pathology related to untreated coronary and cerebral vessels, of which target-vessel thrombosis is only a small proportion.

The largest studies come from the cardiology literature, and the largest of these was the CREST trial, ${ }^{5}$ which was methodologically flawed. Not to be confused with the carotid stent-placement trial with the same acronym, the CREST trial retrospectively identified patients who developed this complication while undergoing coronary intervention (30 cases among 5355). Ten of these 30 patients were then excluded for various reasons. The remaining 20 patients had platelet reactivity assessed anywhere between 4 and 422 days after the episode of thrombosis. No assessment of compliance with medication was performed. Compared with the nonthrombosis group, the thrombosis group had significantly longer lesions treated and lower ejection fractions and were more likely to receive a bare metal rather than drug-eluting stent; these have all been shown by a separate meta-analysis to be independent predictors of stent thrombosis themselves. ${ }^{6}$

Various other trials that also showed a relationship between platelet-function test results and the incidence of target-vessel thrombosis are not particularly compelling due to similarly flawed methodology, ${ }^{7}$ small sample size, ${ }^{8}$ or limited applicability, due to sample populations being confined to either extremely high-risk patients ${ }^{9}$ or to those with drug-eluting stents. ${ }^{10-12} \mathrm{~A}$ more recent well-designed prospective trial by Marcucci et $\mathrm{al}^{13}$ found no significant relationship between high platelet reactivity and stent thrombosis in patients with coronary stents. The most recent GRAVITAS trial (discussed in more detail below) also failed to show a significant relationship. ${ }^{14}$ The afore mentioned POPULAR trial ${ }^{4}$ showed that among all the methods of plateletfunction testing assessed, only light transmittance aggregometry testing, which is highly impractical for routine clinical situations, was correlated with an increased incidence of stent thrombosis and that this relationship was weaker than that demonstrated for other ischemic outcomes.

Before the publication of the articles by Goh et $\mathrm{al}^{1}$ and Fifi et $\mathrm{al}^{2}$ in this month's journal, there was only 1 study in the literature describing a relationship between elevated residual platelet reactivity and an increased incidence of target-vessel thrombosis in neurovascular patients, ${ }^{15}$ but it included only 50 patients, and all were being treated for supra-aortic arteriosclerotic lesions. The data from Fifi et $\mathrm{al}^{2}$ are potentially more convincing, but again, most patients were being treated for atherosclerotic disease and most of the thrombotic episodes were in these patients. As such, the results may not be generalizable to patients with aneurysms. Therefore, the use of platelet-function testing may be justifiable in atheropathic cardiology patients (and patients undergoing carotid stent placement), who stand to have an overall mortality and morbidity benefit separate from that associated with reduced stent thrombosis. However, in the (generally) younger patient with (generally) fewer risk factors for ischemia undergoing aneurysm treatment, the overall mortality benefit is likely to be reduced. This benefit may, in turn, be outweighed by the increased risk of bleeding that inevitably comes with increased platelet inhibition, ${ }^{16}$ as demonstrated by Goh et al. ${ }^{1}$

\section{Assumption 2}

The relationship between high in vitro platelet reactivity and adverse outcomes in cardiology patients can be extrapolated to neurovascular patients. Aside from the questionable assumption of a correlation between reactivity and the specific outcome of targetvessel thrombosis, the automatic extrapolation of results from cardiology patients to neurovascular patients should be scrutinized.

Cardiology patients are far more likely to have presented with acute ischemia and are more likely to be older, to have diabetes, and to smoke cigarettes. All of these may confound both in vitro platelet reactivity and in vivo sensitivity to that reactivity. ${ }^{17-19}$ The discrepant results of studies comparing platelet-function test results with the incidence of stent thrombosis mentioned above may be explained by the fact that the predictive values of these tests are greatest in high-risk patients; this category generally does not include neurovascular patients. It also seems reasonable that cardiology patients will be more likely to be coadministered drugs (statins or proton pump inhibitors) that inhibit the hepatic production of the active metabolite of clopidogrel; a poor response to clopidogrel may, therefore, be less prevalent and less severe among neurovascular patients, reducing the need for testing. Although the important results of Fifi et $\mathrm{al}^{2}$ suggest that high ontreatment platelet reactivity predicts an increased incidence of complications in our patient population, further trials with subgroup analysis of patients undergoing stent placement for aneurysm treatment are required.

\section{Assumption 3}

Increasing antiplatelet pharmacotherapy reduces the incidence of adverse thrombotic events. Numerous studies have shown that various laboratory measurements of high on-treatment platelet reactivity can be reduced, either by increasing the clopidogrel dose $^{20-22}$ or switching treatment to prasugrel, ${ }^{23}$ cilostazol, ${ }^{24,25}$ ticlopidine, ${ }^{26}$ or ticagrelor. ${ }^{27}$ The natural assumption was that similar alterations of pharmacotherapy in patients with high residual platelet reactivity would result in a reduction in the incidence of thrombotic events.

Initial small cardiology trials setting out to test this hypothesis $^{28-30}$ suggested promising results, so several large prospective randomized trials were planned to prove that platelet-function testing could guide pharmacotherapy. The results of the first of these trials were recently published by the GRAVITAS investigators. ${ }^{14}$ The results they obtained, in $>2000$ randomized patients, were uniformly dismal, with no significant improvement in any clinical outcome, despite a significant reduction in platelet reactivity. A second trial, TRIGGER-PCI, was halted by its pharmaceutical sponsor for futility, after a preliminary analysis showed an unexpectedly low event rate. ${ }^{31}$ A recent smaller study of 800 patients demonstrated a reduction in the rates of in-stent thrombosis when patients with high on-treatment platelet reactivity re- 
ceived repeated loading doses of antiplatelet drugs. ${ }^{32}$ Further small studies of approximately 200-300 subjects have subsequently been performed, by using various different tests of platelet function and various alterations to treatment in poor responders, and have shown some success. ${ }^{33-36}$ A recent meta-analysis ${ }^{37}$ of some of these trials demonstrated an overall reduction in mortality and stent thrombosis with modification of antiplatelet therapy on the basis of platelet function testing, though the tests and modifications used were disparate and the overall benefit was highly dependent on the background risk of stent thrombosis (which may be lower in neurovascular patients).

With Fifi et $\mathrm{al}^{2}{ }^{2}$ reporting only a nonsignificant trend to a reduced thromboembolic complication rate with platelet function testing-guided manipulation of pharmacotherapy, the evidence for this practice remains inconclusive. Intrinsically high platelet reactivity may cause, or be associated with, an increased risk of thrombotic complications that cannot be completely or safely nullified, regardless of manipulation of pharmacotherapy or improvement of in vitro test results.

In any case, these trials are fundamentally flawed in that they only assessed the effects of increasing platelet inhibition in clopidogrel-resistant patients, without a control group of normal responders. This omission ignores the previously tested ${ }^{38,39}$ possibility that all patients may have benefited from increased inhibition, regardless of the results of platelet-function testing. Indeed, the OASIS-7 trial $^{40}$ demonstrated that double-dose clopidogrel reduces the incidence of stent thrombosis and other ischemic events in unselected patients, further highlighting this design weakness. Of course, as Goh et $\mathrm{al}^{1}$ have demonstrated, increasing platelet inhibition comes with increased bleeding risks, which may not be acceptable in neurovascular patients with comparatively lower thrombotic risk.

\section{Assumption 4}

A reduction in the incidence of adverse thrombotic events could not be achieved more simply, more cheaply, or more effectively in some other way that does not involve platelet-function testing. Even if, at some point in the future, a randomized prospective trial demonstrates a benefit to altering antiplatelet pharmacotherapy according to the result of platelet-function tests, this would not automatically make it best practice. The cost, inconvenience, and any possible delay of endovascular treatment associated with testing must be compared with other methods of reliable platelet inhibition, as must the relative efficacy of the various methods.

Already, there is substantial evidence to support the routine use of prasugrel (which has the same mechanism of action as clopidogrel but with greater and more predictable inhibition due to improved pharmacokinetics) instead of clopidogrel in all patients undergoing stent placement. ${ }^{41-43}$ The cost of prasugrel may be comparable with the combined cost of clopidogrel and platelet-function testing; and the increased risk of bleeding seen with prasugrel may be mainly or wholly due to reduced platelet inhibition in clopidogrel poor responders (which is the very phenomenon that needs to be overcome). ${ }^{44}$ An identical case could be made for the routine use of ticagrelor or cangrelor in place of clopidogrel, with the addition of adenosine-mediated benefits that neither clopidogrel nor prasugrel can provide. ${ }^{45,46}$

A case can also be made for triple antiplatelet therapy: cilostazol has been shown to enhance the platelet inhibition of clopidogrel in unselected patients. ${ }^{25,47}$ Furthermore, patients with anemia have been shown to have significantly higher on-treatment platelet reactivity than those with normal hemoglobin levels; testing for and treatment of anemia may be a safe and effective way to enhance antiplatelet therapy, with additional health benefits. ${ }^{48}$

Finally and perhaps most simply, much of the problem of elevated residual on-treatment platelet reactivity could be overcome by increasing the maintenance dose of clopidogrel in all patients undergoing endovascular procedures, irrespective of test results, as demonstrated in OASIS-7. ${ }^{40}$

\section{Which Test to Choose: What to Do with the Results}

The ideal test for platelet function should be the test that best predicts which patients will have an improved clinical outcome from tailored pharmacotherapy. As discussed above, the only large well-controlled trial to assess this (GRAVITAS) found no improvement in outcomes when the pharmacotherapy was altered on the basis of the results of the VerifyNow P2Y12 test (Accumetrics, San Diego, California). ${ }^{14}$ VerifyNow is, however, the point-of-care test that appears to best correlate with poor outcome, ${ }^{4}$ despite the fact that its correlation with the labor-intensive platelet function tests that are not currently practical in clinical practice (such as lymphotoxin- $\alpha$ [LTA]) and vasodilator-stimulated phosphoprotein phosphorylation [VASP]) is modest. ${ }^{49}$ The smaller trials showing success from tailored pharmacotherapy have used various different tests, including VerifyNow, LTA/ VASP, and Multiplate Analyzer (Roche, Indianapolis, Indiana). One of these may be proved superior to the others, but the point is currently moot: OASIS $-7^{40}$ demonstrates that evaluating the results of the platelet-function tests that one administers may be a redundant step because it is possible that all patients could benefit from an increased clopidogrel dose or the use of a different antiplatelet agent, regardless of test results. Furthermore, the optimal level of platelet inhibition is yet to be clearly established. Goh et $\mathrm{al}^{1}$ have shown that as platelet inhibition is increased, bleeding risk is increased, and the risk-benefit relationship is likely to be heterogeneous between patient groups.

\section{Summary}

Although the concept of individually tailored antiplatelet pharmacotherapy was conceptually appealing, the evidence for its use is, to say the least, unconvincing. This evidentiary weakness is especially dire for neurovascular procedures because the vascular pathology, the devices used, and the type of patients treated are so different from those assessed in the cardiology-dominated literature. Aside from the lack of sufficient evidence to support the notion that altering pharmacotherapy regimens based on the results of platelet function assays improves clinical outcomes, pharmacology (and pharmaceutical company trial money) has moved beyond clopidogrel to the next generation of more reliable antiplatelet agents; the results of any further trials are likely to be "too little, too late." 


\section{Key Points}

1) Evidence for a relationship between platelet-function-testing results and the incidence of target vessel thrombosis is weak.

2) Almost all of the evidence in favor of such a relationship is found in the cardiology literature, which cannot be automatically extrapolated to neurovascular patients, pathology, and procedures, for a number of reasons.

3) Prospective, randomized, double-blind trials have failed to show any clinical benefit from altering pharmacotherapy based on the results of platelet function tests.

4) There are cheaper, faster, and more effective methods to improve inhibition of platelet aggregation and reduce adverse thrombotic events already available.

\section{REFERENCES}

1. Goh C, Churilov L, Mitchell P, et al. Clopidogrel hyper-response and bleeding risk in neurointerventional procedures. AJNR Am J Neuroradiol 2013;34:721-26

2. Fifi JT, Brockington C, Narang J, et al. Clopidogrel resistance is associated with thromboembolic complications in patients undergoing neurovascular stenting. AJNR Am J Neuroradiol 2013;34:716-20

3. Combescure C, Fontana P, Mallouk N, et al. Clinical implications of clopidogrel non-response in cardiovascular patients: a systematic review and meta-analysis. J Thromb Haemost 2010;8:923-33

4. Breet N, van Werkum J, Bouman $\mathrm{H}$, et al. Comparison of platelet function tests in predicting clinical outcome in patients undergoing coronary stent implantation. JAMA 2010;303:754-62

5. Gurbel P, Bliden K, Samara W, et al. Clopidogrel effect on platelet reactivity in patients with stent thrombosis: results of the CREST study. J Am Coll Cardiol 2005;46:1827-32

6. Iakovou I, Schmidt T, Bonizzoni E, et al. Incidence, predictors, and outcome of thrombosis after successful implantation of drug-eluting stents. JAMA 2005;293:2126-30

7. Ajzenberg N, Aubry P, Huisse M, et al. Enhanced shear-induced platelet aggregation in patients who experience subacute stent thrombosis: a case-control study. J Am Coll Cardiol 2005;45:1753-56

8. Müller I, Besta F, Schulz C, et al. Prevalence of clopidogrel nonresponders among patients with stable angina pectoris scheduled for elective coronary stent placement. Thromb Haemost 2003;89: $783-87$

9. Blindt R, Stellbrink K, de Taeye A, et al. The significance of vasodilator-stimulated phosphoprotein for risk stratification of stent thrombosis. Thromb Haemost 2007;98:1329-34

10. Buonamici P, Marcucci R, Migliorini A, et al. Impact of platelet reactivity after clopidogrel administration on drug-eluting stent thrombosis. J Am Coll Cardiol 2007;49:2312-17

11. Price M, Endemann S, Gollapudi R, et al. Prognostic significance of post-clopidogrel platelet reactivity assessed by a point-of-care assay on thrombotic events after drug-eluting stent implantation. Eur Heart J 2008;29:992-1000

12. Sibbing D, Braun S, Morath T, et al. Platelet reactivity after clopidogrel treatment assessed with point-of-care analysis and early drug-eluting stent thrombosis. J Am Coll Cardiol 2009;53:849-56

13. Marcucci R, Gori A, Paniccia R, et al. Cardiovascular death and nonfatal myocardial infarction in acute coronary syndrome patients receiving coronary stenting are predicted by residual platelet reactivity to ADP detected by a point-of-care assay. Circulation 2009;119:237-42

14. Price M, Berger P, Teirstein P, et al. Standard vs high dose clopidogrel based on platelet function testing after percutaneous coronary intervention. JAMA 2011;305:1097-105

15. Müller-Schunk S, Linn J, Peters N, et al. Monitoring of clopidogrelrelated platelet inhibition: correlation of nonresponse with clinical outcome in supra-aortic stenting. AJNR Am J Neuroradiol 2008;29:786-91
16. Depta J, Fowler J, Novak E, et al. Clinical outcomes using a platelet function-guided approach for secondary prevention in patients with ischemic stroke or transient ischemic attack. Stroke 2012;43: 2376-81

17. Gawaz M, Neumann F, Ott I, et al. Platelet function in acute myocardial infarction treated with direct angioplasty. Circulation 1996;93:229-37

18. Angiolillo D, Fernandez-Ortiz A, Bernardo E, et al. Platelet function profiles in patients with type 2 diabetes and coronary artery disease on combined aspirin and clopidogrel treatment. Diabetes 2005; 54:2430-35

19. Goto S, Sakai H, Goto M, et al. Enhanced shear-induced platelet aggregation in acute myocardial infarction. Circulation 1999; 99:608-13

20. Angiolillo D, Costa M, Shoemaker S, et al. Functional effects of high clopidogrel maintenance dosing in patients with inadequate platelet inhibition on standard dose treatment. Am J Cardiol 2008;101: $440-45$

21. Aleil B, Jacquemin L, De Poli F, et al. Clopidogrel $150 \mathrm{mg} /$ day to overcome low responsiveness in patients undergoing elective percutaneous coronary intervention: results from the VASP-02 randomized study. JACC Cardiovasc Interv 2008;1:631-38

22. Matetzky S, Fefer P, Shenkman B, et al. Effectiveness of reloading to overcome clopidogrel nonresponsiveness in patients with acute myocardial infarction. Am J Cardiol 2008;102:524-29

23. Brandt J, Payne C, Wiviott S, et al. A comparison of prasugrel and clopidogrel loading doses on platelet function: magnitude of platelet inhibition is related to active metabolite formation. Am Heart $J$ 2007;153:66.e9-16

24. Jeong $\mathrm{Y}$, Lee $\mathrm{S}$, Choi B, et al. Randomized comparison of adjunctive cilostazol versus high maintenance dose clopidogrel in patients with high post-treatment platelet reactivity: results of the ACCELRESISTANCE randomized study. I Am Coll Cardiol 2009;53: 1101-09

25. Angiolillo D, Capranzano P, Goto S, et al. A randomized study assessing the impact of cilostazol on platelet function profiles in patients with diabetes mellitus and coronary artery disease on dual antiplatelet therapy: results of the OPTIMUS-2 study. Eur Heart $J$ 2008;29:2202-11

26. Campo G, Vagimigli M, Gemmati D, et al. Poor responsiveness to clopidogrel: drug-specific or class-effect mechanism? Evidence from a clopidogrel-to-ticlopidine crossover study. J Am Coll Cardiol 2007;50:1132-37

27. Gurbel P, Bliden K, Antonino M, et al. Abstract 5901: the effect of ticagrelor in stable coronary artery disease patients non responsive to clopidogrel: the RESPOND study. Circulation 2009;120:S1173

28. Valgimigli M, Campo G, de Cesare N, et al. Intensifying platelet inhibition with tirofiban in poor responders to aspirin, clopidogrel, or both agents undergoing elective coronary intervention: results from the double-blind, prospective, randomized tailoring treatment with tirofiban in patients showing resistance to aspirin and/or resistance to clopidogrel study. Circulation 2009;119: 3215-22

29. Cuisset T, Frere C, Quilici J, et al. Glycoprotein IIb/IIIa inhibitors improve outcome after coronary stenting in clopidogrel nonresponders: a prospective, randomized study. JACC CardiovasC Interv 2008;1:649-53

30. Bonello L, Camoin-Jau L, Arques S, et al. Adjusted clopidogrel loading doses according to vasodilator-stimulated phosphoprotein phosphorylation index decreased rate of major adverse cardiovascular events in patients with clopidogrel resistance. J Am Coll Cardiol 2008;51:1404-11

31. Wood S. TRIGGER-PCI halted: Low events stymie plateletreactivity trial with prasugrel, clopidogrel. www.theheart.org/article/ 1200057.do. Accessed December 6, 2012

32. Siller-Matula J, Francesconi M, Dechant C, et al. Personalized antiplatelet treatment after percutaneous coronary intervention: the MADONNA study. Int J Cardiol 2012 May 30 [Epub ahead of print] 
33. Aradi D, Rideg O, Vorobcsuk A, et al. Justification of $\mathbf{1 5 0} \mathbf{~ m g ~ c l o p i - ~}$ dogrel in patients with high on-clopidogrel platelet reactivity. Eur J Clin Invest 2012;42:384-92

34. Ari H, Ozkan H, Karacinar A, et al. The EFFect of hIgh-dose ClopIdogrel treatmENT in patients with clopidogrel resistance (The EFFICIENT Trial). Int J Cardiol 2012;157:374-80

35. Wang $X$, Zhang D, Zhuang S, et al. Modifying clopidogrel maintenance doses according to vasodilator-stimulated phosphoprotein phosphorylation index improves clinical outcome in patients with clopidogrel resistance. Clin Cardiol 2011;34:332-38

36. Hazarbasanov D, Velchev V, Finkov B, et al. Tailoring clopidogrel dose according to multiple electrode aggregometry decreases the rate of ischemic complications after percutaneous coronary intervention. J Thromb Thrombolysis 2012;34:85-90

37. Aradi D, Komocsi A, Price M, et al. Efficacy and safety of intensified antiplatelet therapy on the basis of platelet reactivity testing in patients after percutaneous coronary intervention: systematic review and meta-analysis. Int J Cardiol 2012 Jun 15 [Epub ahead of print]

38. Patti G, Colonna G, Pasceri V, et al. Randomized trial of high loading dose of clopidogrel for reduction of periprocedural myocardial infarction in patients undergoing coronary intervention: results from the ARMYDA-2 (Antiplatelet therapy for Reduction of Myocardial Damage during Angioplasty) study. Circulation 2005;111: 2099-106

39. Montalescot G, Sideris G, Meuleman C, et al. A randomized comparison of high clopidogrel loading doses in patients with non-ST-segment elevation acute coronary syndromes: the ALBION (Assessment of the Best Loading Dose of Clopidogrel to Blunt Platelet Activation, Inflammation and Ongoing Necrosis) trial. J Am Coll Cardiol 2006;48:931-38

40. Mehta S, Tanquay, Eikelboom J, et al. Double-dose versus standarddose clopidogrel and high-dose versus low-dose aspirin in individuals undergoing percutaneous coronary intervention for acute coronary syndromes (CURRENT-OASIS 7): a randomized factorial trial. Lancet 2010;376:1233-43
41. Wiviott S, Braunwald E, McCabe C, et al. Prasugrel versus clopidogrel in patients with acute coronary syndromes. $N$ Engl J Med 2007;357:2001-15

42. Trenk D, Stone G, Gawaz M, et al. A randomized trial of prasugrel versus clopidogrel in patients with high platelet reactivity on clopidogrel after elective percutaneous coronary intervention with implantation of drug-eluting stents: results of the TRIGGER-PCI study. J Am Coll Cardiol 2012;59:2159-64

43. Capranzano P, Tamburino C, Capodanno D, et al. Platelet function profiles in the elderly: results of a pharmacodynamics study in patients on clopidogrel therapy and effects of switching to prasugrel 5 mg in patients with high platelet reactivity. Thromb Haemost 2011;106:1149-57

44. Dalén M, van der Linden J, Lindwall G, et al. Correlation between point-of-care platelet function testing and bleeding after coronary artery surgery. Scand Cardiovasc J 2012;46:32-38

45. Wallentin L, Becker R, Budaj A, et al. Ticagrelor versus clopidogrel in patients with acute coronary syndromes. N Engl J Med 2009;361: 1045-57

46. Angiolillo D, Schneider D, Bhatt D, et al. Pharmacodynamic effects of cangrelor and clopidogrel: the platelet function substudy from the cangrelor versus standard therapy to achieve optimal management of platelet inhibition (CHAMPION) trials. J Thromb Thrombolysis 2012;34:44-55

47. Lee S, Park S, Hong M, et al. Triple versus dual antiplatelet therapy after coronary stenting: impact on stent thrombosis. J Am Coll Cardiol 2005;46:1833-37

48. Toma C, Zahr F, Moquilanski D, et al. Impact of anemia on platelet response to clopidogrel in patients undergoing percutaneous coronary stenting. Am J Cardiol 2012;109:1148-53

49. Gaglia M, Torguson R, Pakala R, et al. Correlation between light transmission aggregometry, VerifyNow P2Y12, and VASP-P platelet reactivity assays following percutaneous coronary intervention. J Interv Cardiol 2011;24:529-34 